\title{
Comentario crítico sobre el concepto de autopoiesis y la concepción sobre la educación de Humberto Maturana
}

\author{
Rina Pedrol Troiteiro
}

\begin{abstract}
Resumen
En este artículo se analiza el concepto de autopoiesis de Humberto Maturana teniendo en cuenta los elementos que contiene, sus aspectos novedosos y aquellos ya previamente definidos en el campo de la Biología; así como las implicaciones de extender esta interpretación más allá del marco biológico, concretamente en relación a las propiedades de las formas iniciales de vida. Se critica el enfoque biologicista de su concepción y la falta de congruencia entre este concepto y las concepciones que posteriormente están presentes en los principios de la enseñanza que Maturana propone, donde sin abandonar el enfoque biologicista sostiene ideas que pueden resultar atractivas, pero muy abstractas y limitadas al plano teórico.
\end{abstract}

Palabras-clave: Evolución (biología), desarrollo, educación.

\section{Critical comment on Humberto Maturana's concept of autopoiesis and education.}

\begin{abstract}
In this article we analyze Humberto Maturna's autopoiesis concept taking into consideration the elements it contains, its new aspects and those previously defined in the biological field. We also pay attention to the implications of extending this interpretation beyond the biological concepts, that is, in relation with the initials forms of life. We criticize the biological approach and its lack of correspondence to what Maturana proposes. Without abandoning the biological support we can develop theories which could be more attractive and less abstract.
\end{abstract}

Keywords: Evolution (biology), development, education.

\section{Comentário crítico sobre o conceito de autopoiesis e a concepção sobre a educação de Humberto Maturana}

\section{Resumo}

Este artigo apresenta uma análise do conceito de autopoiesis de Humberto Maturana levando em conta os elementos que contém: aspectos novos e aqueles já previamente definidos no campo da Biologia; bem como as implicações de ampliar essa interpretação para além do marco biológico, especificamente em relação às propriedades das formas iniciais de vida. Critica-se o enfoque biologicista de sua concepção e a falta de congruência entre este conceito e as concepções que, posteriormente, estão presentes nos princípios do ensino que Maturana propõe, nos quais sem abandonar o enfoque biologicista sustenta idéias que podem resultar atraentes, sendo, porém, muito abstratas e limitadas ao plano teórico.

Palavras-Chave: Evolução (biologia), desenvolvimento, educação. 


\section{Introdución}

El concepto de autopoiesis ha tenido un importante impacto entre los estudiosos de las diferentes tendencias que se mueven dentro del llamado pensamiento complejo y quizás en buena medida esté avalado por el hecho de que es realizado por Humberto Maturana, biólogo de formación, que adquirió determinado renombre en la esfera de las neurociencias.

Antes de continuar quiero decir que mi primer acercamiento a este concepto fue preparando un curso sobre teorías evolucionistas y que mi primera impresión fue de que se refería más al origen de la vida, que a la evolución propiamente dicha.

Debo reconocer que entre los biólogos, al menos los que yo conozco, no se emplea este concepto y en la mayor parte de los casos, ni siquiera se conoce, aunque los aspectos que en él analiza sean hartamente conocidos y estudiados por otros especialistas.

Quisiera, sin ánimo de alargar demasiado este trabajo, presentar las ideas fundamentales en las que se sustenta este término, para que le quede claro al lector a qué me refiero en el análisis de los diferentes conceptos. ${ }^{1}$

La autopoiesis como teoría fundamental de la vida, plantea que partiendo de un estado primitivo abiótico del planeta Tierra, existían diferentes tipos de moléculas. Algunas de ellas tenían la propiedad de "clausurarse" frente al medio ambiente. Este hecho fue posible mediante la generación de unas estructuras moleculares, a la vez englobantes y aislantes, a partir de lo que ahora llamamos membranas, suficientemente estables y dotadas de plasticidad.

Este tipo de moléculas -y sólo este- fue capaz de formar barreras efectivas y, al mismo tiempo, disponer de la capacidad de realizar intercambios con el entorno para difundir átomos y/o moléculas cargadas eléctricamente, los iones, durante tiempos relativamente largos con respecto a las velocidades moleculares. A este tipo molecular -o macromolecular- pertenecen las proteínas cuya flexibilidad y capacidad de asociación es prácticamente ilimitada. Cuando en la historia del planeta Tierra se dieron las condiciones para la formación de este tipo de moléculas, surgió la vida.

El punto decisivo que ha permitido trazar un límite entre la continua producción molecular abiógena -todavía sin las características que definen "lo vivo"- que se llevaba a cabo en la superficie de los mares y en la atmósfera, y la irrupción de lo viviente, estriba en que hubo momentos en los que fue posible la formación de cadenas de reacciones moleculares de un tipo peculiar. Esta peculiaridad, en la que reside la clave del asunto, es denominada por Maturana (1990, 1997), Autopoiesis. Esta denominación sirve para describir un fenómeno radicalmente circular por el que las moléculas orgánicas forman redes de reacciones que producen a las mismas moléculas que las integran. Tales redes e interacciones que se producen a sí mismas y especifican sus propios límites, no son sino seres vivos.

1 Tomado del artículo: El Tesoro de la alfombra mágica. Los holones se autopreservan, autoadaptan, autotrascienden y se autodiluyen de Javier Del Arco.
Definiríamos, siempre según Maturana, los seres vivos como aquellos entes que se producen a sí mismos y la organización que los define la llamamos autopoiética. Todo sistema autopoiético goza de cinco propiedades importantes, denominadas: Autonomía, Emergencia, Clausura de Operación, Autoconstrucción de Estructuras y Reproducción autopoiética.

\section{Autonomía:}

La célula pone de manifiesto la superación de la correspondencia, punto por punto, con respecto al medio ambiente. Ella exige una perspectiva de autonomía, en el sentido que la célula requiere de la creación a distancia con respecto al mundo circundante y esto significa que sólo desde la perspectiva celular se puede determinar lo que es relevante y sobre todo lo que le es indiferente. Ejemplo de ello es el transporte activo y selectivo a través de las membranas.

\section{Emergencia:}

La aparición de un orden cualitativo en la célula, distinto al de las moléculas que integran un mineral, no puede deducirse a partir de sus características materiales o energéticas. La emergencia señala la aparición de un orden nuevo, cuyas características solo pueden ser advertidas después de su constitución. Lo que es emergente en la célula es su "operación", la forma en cómo están organizadas y cómo esa organización se lleva a efecto.

\section{Clausura de operación:}

Los sistemas autopoiéticos son sistemas cuya operatividad es cerrada y cuyos componentes son productos en el interior de un retículo clausurado. Clausura no debe entenderse aquí como lo opuesto a apertura, sino como la condición de su posibilidad. Lo que está clausurado en la autopoiesis es el control mismo mediante el cual los elementos se organizan de manera emergente. La clausura operacional supone que las células produzcan operaciones exclusivas reproductoras de vida por las que se mantienen en la vida, es decir, no existe otra cosa que su propia operación.

\section{Autoconstrucción de estructuras:}

Dado que la operación en una célula está, en un determinado momento clausurada, no puede captar estructuras: ella misma debe construirlas. Por lo tanto el proceso de autoconstrucción deberá entenderse, como producción de estructuras propias, mediante operaciones propias. Los sistemas clausurados en su operación producen sus propios elementos y sus propios cambios estructurales. No existe, ninguna operación causal del entorno del sistema, sin que el mismo sistema la provoque: todo cambio de estructura, trátese de adaptación o rechazo, es en última instancia, autoinducido. 


\section{Reproducción autopoiética:}

El estado siguiente de un sistema está determinado a partir de la estructuración anterior a la que llegó la operación (Del Arco, 2007, pp. 2-3)

Como señalé anteriormente estas explicaciones se refieren a la etapa de tránsito de lo abiótico a lo biótico ${ }^{2}$, por cierto sin analizar las condiciones, los lugares y los múltiples elementos que participaron en este proceso ${ }^{3}$, así como las posteriores etapas que dieron lugar a la rica $y$ compleja diversidad de lo vivo, a partir del surgimiento de los eucariotas hace 1,200 millones de años. Por ello este concepto, como teoría evolucionista, realmente no responde a las expectativas biológicas actuales, que son de explicar no sólo el qué, sino también el cómo ocurrió el proceso de origen y evolución de la vida, que es en última instancia lo que hoy en día está sometido a amplios debates.

Las etapas para llegar a la formación de la vida y las moléculas primero y las estructuras después que participan en el proceso, están incluidas en todas las teorías evolucionistas que sustentan el origen abiogénico de la vida, aunque los diferentes autores empleen otros términos, pero son las mismas ideas.

Ante esto, cabría preguntarse por qué han resultado atrayentes estos planteamientos, si las propiedades a que se hace referencia tampoco son nuevas, pues se han definido desde la primera mitad del siglo $\mathrm{XX}$, con teoría como la de A. Oparin en 1938 y se corresponden con las cuatro características esenciales de la vida: la delimitación de un espacio interno, el intercambio selectivo con el medio, la existencia de una fuente energética propia, a partir del acoplamiento de reacciones internas y la reproducción de sus características, a través de los mecanismos de la herencia.

En mi criterio, lo interesante de este concepto y lo que lleva implícito, es su formulación con un alto grado de generalización, resaltando lo esencial del proceso y con un carácter más filosófico y con la posible intención de ser generalizable a otros sistemas, aunque esto puede resultar a su vez peligroso, pues puede llevar los análisis a un plano reduccionista. Esta forma de definir las propiedades de este sistema, no deja de ser tentadora por su novedad para otros contextos y porque emplea un lenguaje afín al pensamiento complejo.

Pero, sin negar la utilidad de estas formulaciones creo que llevan implícito, quizás por la forma en que se realiza su generalización, algunas omisiones y situaciones que

2 Etapa clave en el origen de la vida, que se estima que ocurrió hace 4,100 millones de años, en la que se pasó de la existencia de moléculas orgánicas, pero sin las propiedades de la vida, a las primeras formas de vida denominadas protobiontas que aún no poseen todas las propiedades esenciales de ésta.

3 Tales como las fuentes energéticas, el tránsito y las características reactivas de las diferentes atmósferas primitivas, el origen de las moléculas orgánicas, los movimientos de la corteza terrestre, el régimen de precipitaciones, etc. y el efecto sinergético de todos estos factores pueden generar algunos errores de interpretación y talvez, el riesgo mayor que va más allá de omisiones o valoraciones que es el tratar de extrapolar estas explicaciones al plano de las relaciones sociales y de las características del hombre, olvidando o no asumiendo que éste ya ha dejado de ser un ser eminentemente biológico para adquirir una dimensión mucho más compleja donde están incluidos sus componentes psíquicos, sociales y culturales.

Si me concentro en lo propiamente biológico de las explicaciones, para mi gusto, deja demasiado implícito, la naturaleza de la interacción con el ambiente, ya que no se menciona en ningún momento su importancia, a no ser como suministrador de recursos; pues si bien el final de los procesos es la conservación de las características esenciales de la vida, éstas sólo se logran en interacción dinámica con el medio, de forma que la respuesta concreta que se obtiene es cambiante en el tiempo y en cada contexto y es modulada por la interrelación de todos los elementos que lo constituyen, tanto internos como externos. Por tanto, el ambiente no sólo es suministrador de recursos, sino parte del sistema de control del organismo vivo y a su vez, un componente que recibe el efecto modificador de éste.

Además, me parece totalmente inapropiado utilizar el término de clausura, que significa lo mismo que cerrado, pero con un sentido mas definitivo, para después decir que eso no significa que no haya algún tipo de intercambio con el medio, entonces es introducir un término nuevo, pero totalmente ambiguo; cuando este proceso está adecuadamente explicado incluyendo la importante dinámica que este conlleva; ejemplos de las diferentes formas en que estas propiedades han sido analizadas desde principios del siglo pasado, se pueden leer en el artículo de Garbisu, Amézaga, Albizu y Alkorta (2003), "La esencia de los seres vivos".

Por supuesto, que yo puedo centrarme en lo esencial, que es que se conserva la vida, pero al no dejar clara la vía, no mencionar la complejidad en que esto se produce al transponerlo a otros sistemas, puede conducir a un reduccionismo.

Por otra parte, se enfatiza la llamada autorreproducción, que no es más que lo que se explica mediante los mecanismos de la herencia, tan ricamente estudiados desde la mitad del siglo pasado y que ha eliminado de su vocabulario el auto porque simplifica toda la complejidad de sus mecanismos.

En mi criterio esto es más preocupante cuando estos conceptos se emplean en otros sistemas con otros contextos. Por ejemplo el social, ¿valoro el medio como algo fuera del sistema que está delimitado de éste?, ¿que se autorreproduce? Y si analizo la formación de lo psicológico, los productos de la educación, la enseñanza, lo cultural, ¿lo valoro como determinado por características internas biológicas, donde el medio es solo una fuente de suministros de elementos materiales para la construcción de estructuras que son tomadas selectivamente en respuesta a un plan biológico preexistente, predefinido y cerrado a otras influencias?. 
Hay otro aspecto, en mi criterio muy importante, que la autopoiesis no toma en consideración ni en lo biológico ni en la extrapolación a otros sistemas, me refiero a la historicidad de los procesos, lo que el devenir histórico ha permitido obtener y estructurar en los sistemas, me resulta contradictorio pues, no hay evolución sin historia. Incluso la propia historia que se va concretando y estructurando en esta interacción es lo que después forma parte de las nuevas propiedades biológicas (emergentes). Aunque es válido señalar que la precisión que se hace de emergente es muy adecuada: la forma en como están organizadas y cómo esa organización se lleva a efecto.

Estudiando este tema me encontré con esta idea escrita por Del Arco (2007) en el artículo ya nombrado: "El concepto de autopoiesis es clave en la Biología moderna y creemos que es nuestra obligación aclararlo porque además, guarda determinadas relaciones con la Teoría Sociológica nada desdeñables, se esté o no de acuerdo con ellas" (Del Arco, 2007, p.1)

Y precisamente me identifiqué con esta opinión, pues es evidente un acercamiento del concepto de autopoiesis al de la sociobiología ${ }^{4}$, pero ésta como yo la entiendo, es una teoría reduccionista, cientificista y reaccionaria, que minimiza el papel de la sociedad en la formación y desarrollo del ser humano, la importancia de las relaciones sociales, de forma que lo que entendemos por injusticias sociales no serán tales, sino manifestaciones de las diferencias biológicas entre los hombres.

Por supuesto estas ideas no sólo aparecen en la sociobiología, sino que también están presentes, más actualmente, en los trabajos de Jensen (1995) y en el libro de Herrnstein y Murray (1994) "The Bell Curve" cuando plantean:

Desde que la inteligencia y otras habilidades mentales dependen de la estructura fisiológica del cerebro, y desde que el cerebro, como otros órganos, está sujeto a las influencias genéticas, ¿cómo puede dejar de considerarse la obvia probabilidad de la influencia genética en la inteligencia? (Herrnstein \& Murray, 1994, p. 104)

A su vez, estas ideas recibieron una fuerte respuesta de Lewontin, Rose y Kamin (1987) en su libro "No está en los genes", cuando dicen:

Lo biológico y lo social no son ni separables, ni antitéticos, ni alternativos, sino complementarios. (...) Todas las causas del comportamiento de los organismos son en el sentido temporal al que deberíamos limitar el término causa, simultáneamente sociales y biológicos, y todos ellos pueden ser analizados a muchos niveles (...) Todos los fenómenos humanos son simultáneamente sociales, biológicos, del mismo modo que son al mismo tiempo químicos y físicos. (Lewontin \& cols., 1987, p. 343)

4 Sociobiología: teoría, planteada por Edward Wilson en 1929, que trata de explicar todos los fenómenos sociales y psicológicos a partir de las leyes de la herencia y de otras leyes biológicas como las de la evolución, de manera particular: la selección natural.
Si los planteamientos de la autopoiesis se aplican a todos los seres vivos, pero en el caso del hombre, se presentan nuevas propiedades emergentes de sus condiciones sociales y culturales, históricamente constituidas, y su componente psicológico, entonces debemos admitir que el sistema comienza a tener otros componentes que no se ajustan a la clausura de operación, la autoconstrucción de estructuras y la reproducción autopoiética.

Si esta teoría de Maturana, es sólo para explicar el origen de la vida y no su evolución y las propiedades de otros sistemas y para ilustrar a filósofos y estudiosos de la ciencia que existen sistemas, en biología, con características que no se ajustan a lo que esquemáticamente se había entendido hasta ahora, bienvenida sea. Pero cuidado con que en su aplicación, paradójicamente, se niegue la esencia del pensamiento complejo que plantea que las propiedades de los sistemas no pueden ser descritas de forma significativa en base al análisis de sus elementos separados; ya que la comprensión de los sistemas, sólo es posible cuando se estudian globalmente, involucrando todas las interdependencias de los subsistemas.

Por otra parte, no quiero negar que la forma de análisis de los procesos biológicos básicos, incluidos en el concepto de autopoiesis, no tenga una importancia para el pensamiento complejo, atendiendo a los méritos que anteriormente señalé, todo el conocimiento tiene un valor y una utilidad, pero siempre que se emplee teniendo en cuenta su alcance y limitaciones, sus concepciones de partida, es decir la cuota de incertidumbre que le corresponde, por ello su empleo debe ser a partir un análisis crítico de su contenido.

Y es precisamente pensando en su empleo que quiero, a la luz de lo planteado, reflexionar sobre las consecuencias que puede tener en la práctica educativa las concepciones que sustentan esta teoría y que después el propio autor amplía, modifica o refuta, a partir de las concepciones que sobre la educación él tiene. Sus ideas resultan algo análogas a las de Piaget $(1968,1969)$, en el sentido que cuando éste habla del desarrollo psicológico lo considera de una naturaleza más espontánea y madurativa y cuando habla del proceso pedagógico y educativo lo considera más producido y artificial, separando los procesos de aprendizaje y desarrollo o sin considerar a la educación, enseñanza y aprendizaje como componentes estructurales y funcionales del desarrollo psicológico. Esto se hace evidente en Piaget (1968) cuando plantea lo siguiente:

La pedagogía experimental sólo recae, en efecto, en el desenvolvimiento y los resultados de procesos propiamente pedagógicos, lo que no significa como vamos a ver, que la psicología no constituya una referencia necesaria.

Equivale a decir que los problemas planteados son otros y contemplan, antes que los caracteres generales y espontáneos del niño y de su inteligencia, la modificación de éstos mediante el proceso en cuestión. (p. 28)

Si aceptamos que los sistemas se autogeneran, se autorregulan y por tanto se autoconservan, entonces qué 
función le corresponde a la enseñanza en el proceso de desarrollo de los individuos, quizás un mero soporte externo para que el desarrollo se produzca; incluso esta teoría no toma en consideración la importancia de ningún tipo de relaciones sociales. Esto se hace evidente cuando se reduce el desarrollo del individuo a la red de desarrollo biológico de los demás: "La ontogenia individual de todos los organismos participantes ocurre, fundamentalmente, como parte de una red de coontogenias que llevan a la constitución de la unidad de tercer orden." (Maturana \& Varela, 1988, p. 193).

Desarrollo biológico que además, se presenta con una concepción estructuralista que no toma en consideración el funcionamiento del sistema, la complejidad de los vínculos que se establecen y aún cuando se ha planteado que se trata de vínculos entre sistemas cerrados, es muy difícil imaginarse esos vínculos y más aún, comprender la importancia que ellos puedan tener para el desarrollo de cada uno de los elementos de la red.

Si queremos lograr una mejor estructuración del sistema de enseñanza basados en la concepción de que la enseñanza promueve el desarrollo, de poco nos sirven las concepciones que se derivan de la tan renombrada autopoiesis, en la que el desarrollo, empleando un vocabulario postmoderno, está implícito en el sistema, de forma que no se aleja de las concepciones innatistas de Jensen (1995), Herrnstein y Murray (1994) o biologicistas de Piaget (1968, 1969).

La concepción biologicista de Maturana $(1990,1997)$ se evidencia cuando hace referencia a la "biología del conocimiento", o a que "la educación es transmitida de linaje en linaje"; y cuando en su teoría del amor, como fuente de la educación, nos dice:

El amor, como emoción primera conque surge la vida, es la que se ha reproducido desde la familia ancestral. Así, donde aprenden los niños es en el convivir diario y la educación no sería más que la expansión de ese vivir. Somos seres biológicamente amorosos como un rasgo de nuestra historia evolutiva. El amor ha sido la emoción central conservada en la historia evolutiva que nos dio origen desde hace unos cinco a seis millones de años atrás. (Santa Cruz \& Mendoza, 2007, p. 1).

Quizás de forma velada, esta teoría que en primera instancia resulta muy atractiva y sensible no deja de concebir al amor como una emoción biológica que el hombre adquiere en su formación o evolución biológica y se trasmite de generación en generación, no olvidar que emplea el término linaje que implica una herencia biológica.

Así cuando nos habla de la "biología del amor", postula que las emociones se especifican en el curso de las relaciones del organismo "en el medio"5, y de hecho constituyen un factor guía en el devenir de la historia evolutiva de los seres vivos. Es decir que en el mejor de los casos, el medio es un factor guía para seguir un curso que de manera general esta prefijado, e incluso el hecho de que refiere en el medio y no con el medio refuerza esta idea de mero soporte. En este análisis no se insiste en que el amor surge y se forma, mediado también en el ser humano, por el sistema de relaciones sociales e interpersonales y que según el contenido de dichas relaciones (lo cual es producido culturalmente a todo lo largo de la historia de la sociedad, con diferentes formas) se produce amor o violencia, odio o rechazo, entre las personas o los grupos de personas.

No se puede negar que en sus ideas hay afirmaciones hermosas, como cuando dice:

La educación, es un proceso de transformación humana en la convivencia. Con esto queremos decir, que ocurre en todas las dimensiones relacionales del vivir del niño, tanto en los espacios privados de la familia o del jardín infantil, como en los espacios públicos de la calle y el barrio.

La educación es un proceso en el que tanto los niños como los educadores cambian juntos en forma congruente, en tanto permanecen en interacciones recurrentes. De modo que los niños aprenden a vivir en cualquier dominio de vida donde sus maestros los encaminen (Tejeda, 2009, p. 1).

\section{Así también afirma:}

La educación es un proceso de transformación de vida que sigue un curso definido por la manera de vivir de los padres y educadores. En este proceso, el niño se transforma en un tipo de ser humano u otro según el tipo de experiencias vividas recurrentemente con sus padres y educadores. (Tejeda, 2009, p.1)

El propio autor del artículo de estas citas enfatiza:

Nos seduce el pensamiento de Humberto Maturana en sus implicancias para la educación en general y la educación preescolar en particular. La comprensión de lo humano requiere la comprensión de la dinámica biológica que lo origina. Tenemos que entender los fundamentos biológicos del saber y el aprender para expandir nuestra comprensión de lo que ocurre en el proceso educativo y las consecuencias que tiene ese proceso para la vida humana. (Tejeda, 2009, p.1)

Es decir, que hay una aceptación tácita de que es la dinámica biológica la que explica o fundamenta el proceso de enseñanza y no toma en consideración la mediación de la dinámica de lo social y lo cultural en la que vive el sujeto desde que nace y más complejo aún, la mediación compleja de lo biológico, lo social y lo cultural con lo propiamente psicológico que se va conformando. Lo preocupante de este análisis es que se ignora que lo social y lo cultural pueden afectar el desarrollo psicológico humano y la efectividad de los procesos de aprendizaje.

A continuación de estas afirmaciones se refuerza el papel de las emociones y particularmente del amor en esa función educativa, cuando dice: "La historia humana y la de los seres vivos en general, sigue el curso de las emociones (en particular, de los deseos) no el de los recursos o la tecnología." (Tejeda, 2009, p. 2).

5 Comillas de la autora del trabajo 
No obstante estas afirmaciones, no encontramos referencia a los procesos que garantizan el papel dominante de las emociones o los mecanismos biológicos del amor y la enseñanza. Las ideas se quedan a nivel de enunciados generales sin abordar cómo se produce su formación, hace referencia a que los profesores deben tratar a sus educandos con amor, pero no les da ni fundamentos, ni herramientas para construir ese amor.

Lo más cerca que está de una explicación la encontramos cuando hace referencia al papel del lenguaje, con una concepción completamente postmoderna:

Hacemos cosas con nuestros cuerpos (incluyendo el sistema nervioso), y fluimos en el lenguaje en nuestras interacciones diarias. La estructura de nuestros cuerpos cambia según nuestro modo de fluir en el lenguaje (basta mirar la ampliación en el tamaño del cerebro que significó el uso del lenguaje en nuestros primeros antepasados). Nada de lo que hacemos en el lenguaje es irrelevante, porque nos transformamos en nuestros cuerpos según lo que hacemos en el lenguaje, y hacemos en nuestro lenguaje según lo que se transforma en nuestros cuerpos.

A medida que el niño aprende a usar el lenguaje, crea con otros diferentes modos de vida, dado los diferentes hechos en los que participa; y llega a ser en su cuerpo según el uso del lenguaje en el cual crece. Como resultado, cuando adulto, crea el mundo que vive como una expansión del mundo que creó cuando niño (Maturana, 1990, p. 3).

El lenguaje parece ser como una adquisición producto de la evolución biológica humana; esta idea se refuerza cuando dice:

Los linajes se conservan según un modo de vivir, en la reproducción sostenida que se aprende de una generación a otra. Es en el placer de juntarse, en la reunión con los otros donde el niño aprendería a convivir y sólo el lenguaje, como modo de coordinar los haceres y las emociones, permitiría esto. (Tejeda, 2009, p.1)

No obstante estos enfoques, en otras ocasiones Maturana (1997) le atribuye un papel a la educación, que es transformador y desarrollador de la autonomía que resulta inconsistente con las ideas biologicistas que en otras ocasiones plantea, ya que no establece nexos entre la determinación biológica de este proceso de educación y después las posibilidades que éste tiene de lograr en el niño adecuadas capacidades. Asimismo, dice que todos los niños son igualmente inteligentes, que lo que varia es la dosis de amor que reciben; además de una hiperbolización tanto de un componente como del otro, todas estas ideas no parecen formar parte de un cuerpo teórico bien estructurado y fundamentado, más bien bellas ideas lanzadas al viento.

Otro aspecto que se hace evidente de su teoría y en lo que coincide, como ya referimos, con la concepción piagetiana, es el divorcio entre la concepción de desarrollo, que para él solo incluye aspectos biológicos y la enseñanza, la cual, aunque también incluye aspectos biológicos, la refuerza con elementos externos y propiamente psicológicos como las emociones. En Maturana (1990), en todo momento, se hace evidente la falta de una concepción general, integral y suficientemente fundamentada que sirva de soporte y fundamentación a la labor educativa de la escuela y toda la sociedad.

Según mi concepción es necesario que los profesores posean una visión holística de ambos procesos como nos la plantea Guillermo Arias (2004) cuando analiza la zona de desarrollo próximo, desde el enfoque histórico cultural:

El desarrollo y la formación psicológica son contenidos generalizadores de los procesos de aprendizaje y de la enseñanza, en la persona y sólo en la persona, como consecuencia de la acumulación de la experiencia individual que median las vivencias del sujeto. Por ello los procesos de aprendizaje y los procesos vivenciales son los que halan el desarrollo a la vez que dependen del desarrollo y las ayudas, que como se observa son trascendidas por el desarrollo que va ocurriendo, es como si ellos, halaran e irradiaran el contenido del desarrollo psicológico. (Arias, 2004, p. 236)

Aquí Arias (2004) enfatiza la importancia de la vivencia en los procesos de enseñanza y aprendizaje, y, en la siguiente cita podemos ver como Vygotski (1996) la explicaba, refiriendo no sólo su componente biológico; sino también social:

La vivencia posee una orientación biosocial, es algo intermedio, que significa la personalidad con el medio, revela lo que significa el momento dado del medio para la personalidad. La vivencia determina de qué modo influye sobre el desarrollo del niño uno u otro aspecto del medio. (...). Lo esencial no es la situación por sí misma en sus índices absolutos, sino el modo cómo vive dicha situación el niño. (p. 383)

El componente emocional y afectivo no está ausente en este enfoque, todo lo contrario, se plantea que es lo que mueve, estimula y mediatiza la zona de desarrollo próximo, esto puede verse en las siguientes palabras:

Lo que mueve y estimula, lo que mediatiza la dinámica de la zona de desarrollo próximo es lo emocional y lo afectivo. Es tanto así que en estudios con niños en edad preescolar, hemos podido constatar que esta zona se altera, se hace más efectiva o menos efectiva, en dependencia de las relaciones adecuadas o inadecuadas a las que está acostumbrado el niño y la actitud que esto crea, hacia la realización de nuevas tareas. (Arias, 2004, pp. 236-237)

Entonces acudir al amor como fundamento del saber y el aprender no es inapropiado, de hecho podemos verlo desde el punto de vista de las vivencias positivas que producirán en el niño las manifestaciones de amor, el problema está en verlo como rasgos inherentes a las cualidades biológicas del individuo; además de que estas ideas no forman parte de un sistema conceptual que articule la formación psicológica con el desarrollo y los procesos de enseñanza y aprendizaje, es decir que suministre un cuerpo teórico válido para una práctica educativa. 
Es por todo lo planteado, que pienso que las concepciones de Maturana poco nuevo y fructífero pueden aportar a crear una enseñanza desarrolladora que tenga en cuenta las diferencias individuales de los estudiantes, que le dé conocimientos sólidos, en el plano teórico e instrumental, a los profesores para desarrollar su labor y que considere también la relación indisoluble entre dos procesos diferentes como el desarrollo emocional y el intelectual.

Por ello he enfatizado el carácter postmoderno de sus ideas, palabras que suenan bien, que resultan seductoras, pero que están divorciadas de la real problemática que hay que enfrentar, de su dinámica y es por esto, que en mi criterio, tiene muy poca aplicabilidad en la práctica educativa.

\section{Referencias}

Arias, G. (2004). La Persona en lo Histórico Cultural. São Paulo: Linear B.

Del Arco, J. (2007). El Tesoro de la alfombra mágica. Los holones se autopreservan, autoadaptan, autotrascienden y se autodiluyen. Filosofía de la ciencia y la tecnología - Blog de epistemología de Tendencias 21. Recuperado: 01 abr 2007. Disponível: www. tendencias21.net.

Garbisu, C., Amézaga, I., Albizu, I., \& Alkorta, I. (2003). La esencia de los seres vivos. Ecosistemas. Recuperado: 15 jan 2010. Disponível: www.aeet.org/ecosistemas/033/opinion1.htm.

Herrnstein, R. J., \& Murray, C. (1994). The Bell Curve. New York: Free Press Paperback.
Jensen, A. R. (1995). The diferences are real. En N. Glauberman \& R. Jacoby (Eds.), The bell curve debate (pp. 617-629). New York: Times Books.

Lewontin, R. C., Rose, S., Kamin, L. J. (1987). No está en los genes. Barcelona, Espanha: Editorial Crítica.

Maturana, H. (1990). Emociones y lenguaje en educación y política. Santiago: Colección HACHETTE/COMUNICACIÓN - CED.

Maturana, H. (1997). La objetividad. Un argumento para obligar. Santiago: Dolmen.

Maturana, H., Varela, F. (1988). The Tree of Knowledge. Boston: New Science Library.

Piaget, J. (1968). Educación e Instrucción. Buenos Aires: Editorial Proteo S.C.A.

Piaget J. (1969). Psicología y Pedagogía. Barcelona, Espanha: Ediciones Ariel.

Robles, F. (2002). Sistemas de Interacción, Doble Contingencia y Autopoiesis Indexical. Recuperado: 15 jan 2010. Disponível: www.moebio.uchile.cl/15/robles.htm.

Santa Cruz, X., \& Mendoza, J. (2007). Biología del Amor y Educación: Ecoderivando con Humberto Maturana. Ecovisiones.

Tejeda, C. (2009). Humberto Maturana: El amor como propuesta para el cambio. El diario ciudadano de la provincia de Osorno.

Vygotski, L. S. (1996). Obras escogidas (Vol. 4). Madrid: Visor.

Wilson, E. O. (1980). Sociobiología, la nueva síntesis. Barcelona, Espanha: Ediciones Omega.

Recebido em: 06/07/2009

Aprovado em: 09/12/2009

Rina Pedrol Troiteiro (ripetroi@gmail.com)

Universidad de La Habana

Correspondência

Armas No 461 e/ C y D, Lawton, 10 de Octubre.

Ciudad: Habana, Habana 7 Cuba. 